\title{
The Influence of Serum Uric Acid Level on Alzheimer's Disease: A Narrative Review
}

\author{
Mengyuan Qiao $(\mathbb{D}$, Chongli Chen $(\mathbb{D}$, Yuqing Liang $\mathbb{D}$, Yuxi Luo $\mathbb{D}$, and Wenbin Wu \\ Department of Geriatrics, Hospital of Chengdu University of Traditional Chinese Medicine, Chengdu City, Sichuan Province, China
}

Correspondence should be addressed to Wenbin Wu; wwb1201@vip.sina.com

Received 14 February 2021; Revised 21 May 2021; Accepted 27 May 2021; Published 3 June 2021

Academic Editor: Yuzhen Xu

Copyright ( 2021 Mengyuan Qiao et al. This is an open access article distributed under the Creative Commons Attribution License, which permits unrestricted use, distribution, and reproduction in any medium, provided the original work is properly cited.

\begin{abstract}
As a powerful antioxidant in the human body, uric acid (UA) has been the subject of increasing research that focused on its influence on Alzheimer's disease $(\mathrm{AD})$ in recent years. The latest literature was gathered to describe the influence of serum uric acid (SUA) level on the onset and progression of AD and to analyze the possibility that SUA is a biomarker of Alzheimer's disease. A large number of existing studies suggested that the SUA level was lower or tended to decrease in patients with AD, and increased SUA level may have a protective effect in $\mathrm{AD}$, which could reduce the risk of onset and slowing the course of the disease. However, some Mendelian randomization analyses suggested that genetically determined uric acid was not associated with AD risk. Existing research results are contradictory due to the high inconsistency of the studies, the selection of subjects, and other factors. UA also showed a strong association with cognitive function, and there appeared to be a gender-selective neuroprotective action. Due to its potent antioxidant properties, the low uric acid level may contribute to oxidative stress to accelerate disease progression. But some preclinical data showed a possibility that in some special cases, UA had a prooxidant properties. The possibility was raised in the discussion of the underlying mechanism that both the low uric acid level and the rapidly progressive course of the disease were the consequence of malnutrition. This paper reviews recent advances in the study of SUA and AD which offers the possibility of new biomarker, new prevention, and treatment strategies for Alzheimer's disease.
\end{abstract}

\section{Introduction}

Uric acid (UA) is a product of purine metabolism, which is a natural and powerful antioxidant that helps remove superoxide by blocking the degradation of superoxide dismutase, the enzyme responsible for purine removal [1]. The SUA level is altered according to the balance between dietary purine intake, xanthine oxidase activity, and renal UA excretion [2]. When the equilibrium is disturbed, hyperuricemia or hypouricemia occurs. In the past, uric acid was mainly considered to be related to gout. But in recent years, there has been a proliferation of studies between uric acid and neurodegenerative disease, mainly including dementia, Parkinson's disease, amyotrophic lateral sclerosis, and multiple system atrophy.

Current trends indicate that early detection of $\mathrm{AD}$ by noninvasive approaches is a popular area of research. A growing number of studies have demonstrated that UA was linked with the risk, progress, and prognosis of $\mathrm{AD}$, through its neuroprotective effect, antioxidant capacity, metal complexation, and other mechanisms [3]. However, due to the inconsistency of research types and limitations of various research types, existing research results are contradictory. This article reviewed the progress of epidemiological and other studies on the influence of SUA on $\mathrm{AD}$, aiming at exploring the possibility of SUA as a peripheral marker of $\mathrm{AD}$ and providing a reference for future research on peripheral markers of $\mathrm{AD}$.

\section{Method}

On December 04, 2020, we performed a search of PubMed and Web of Science. Language and regional restrictions were not imposed. We searched the database above using search terms including "Alzheimer" AND "uric acid", "dementia" AND "uric acid", and "cognitive" AND "uric acid". The reference lists of included studies and relevant reviews were studied manually to minimize the omission of potentially 
eligible articles. We mainly selected literature from 2016 to 2020 and included older literature that was commonly cited and highly valued (Figure 1).

\section{Level of SUA Was Relevant to the Risk of AD}

In recent years, there has been an increasing interest in studying the risk of developing $\mathrm{AD}$ in populations with different concentrations of SUA. However, the relationship between level of SUA and the risk of AD has been conflicting in different types of studies, and the evidence is weak in elderly subjects.

3.1. Results from Epidemiological Studies. In recent years, several prospective cohort studies with large sample size and long follow-up period have been published. A prospective cohort study in Sweden enrolled a population-based sample of 1462 females who were followed for up to 44 years. During the follow-up period, serum uric acid levels were measured twice. They found that a higher SUA concentration (per standard deviation of $76.5 \mathrm{mmol} / \mathrm{L}$ ) was related to a lower risk for incident dementia $(n=320$; hazard ratio (HR) 0.81 ; confidence interval (CI) $0.72-0.91)$ and both $\mathrm{AD}(n=152$; HR 0.78; CI 0.66-0.91) and vascular dementia (VD; $n=52$; HR 0.66; CI 0.47-0.94) [4]. This finding suggested the protective effect of SUA in the onset of incident dementia regardless of subtype. For the present literature, this cohort had the longest follow-up years and was a strong evidence. However, the limitation was that the sample population included only women. So whether the conclusions are also applicable to men is yet to be confirmed by other studies.

Latourte et al. evaluated the longitudinal link between the level of SUA and incident dementia in a large cohort followed for 12 years. This study enrolled 1598 individuals (mean (SD) age 72.4 (4.1) years, $61.7 \%$ female). The result showed that 110 participants developed dementia. After multivariate adjustment, the multivariate HR of the highest versus lowest SUA levels was 1.79. The correlation was stronger with other subtypes compared to $\mathrm{AD}$, including VD and mixed dementia (MD) [5]. The elderly with a high level of SUA may mark the higher risk of incident dementia, especially VD or MD. The conflicting findings of these two studies may be due to the different gender structures of the participants and the duration of follow-up years.

Because of the difficulty of follow-up and other factors, only a few studies have considered the subtype classification in the cohort study of dementia. Alam et al. studied 11,169 participants in communities (ARIC) cohort with 24.1-year median follow-up period. As the study progressed, a total of 2005 cases of incident dementia were detected. They conducted adjustments including cardiovascular risk factors. After that, SUA and incident dementia showed an uncorrelated outcome (HR, 1.03; 95\% CI, 0.88, 1.21). Elevated baseline levels of SUA were relevant to faster decline of cognition (25-year global $z$-score difference, -0.149 ; 95\% CI, -0.246 , -0.052) [6]. The results of this study may be due to the different influences of SUA on the onset of different subtypes of incident dementia. There was no separate subtype analysis of $\mathrm{AD}$ and $\mathrm{VD}$ in data analysis, which may lead to the cancel-

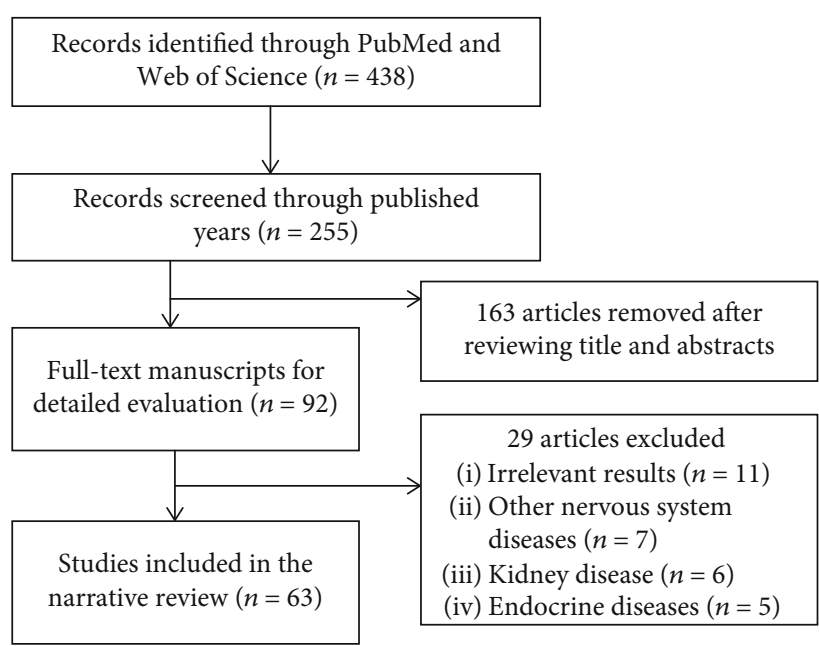

FIgURE 1: A flowchart shows the selection of study in the narrative review, which includes the final results of the initial search.

lation of positive and negative effects, resulting in the final result of no correlation. Another prospective study conducted in the UK without subgroup analysis showed that people in the lowest level group of SUA had a $25 \%$ increased risk of dementia compared to those in the highest SUA level group (HR $=0.75,95 \% \mathrm{CI}$ : 0.64-0.87). SUA was an independent predictor of dementia [7]. These studies did not include the analysis of the subtypes of incident dementia. AD and VD were analyzed together, which would have some impact on the results and conclusions.

Two population-based cohort studies $[8,9]$ indicated that gout was inversely relevant to the onset of $\mathrm{AD}$. Gout patients had lower risks of AD. Among them, Hong et al. [9] took into account the effects of vascular dementia. According to sensitivity analysis in the study, after classifying patients with stroke before $\mathrm{AD}$ as vascular dementia, the conclusion remained the same. However, this inverse association could not be definitively attributed to the neuroprotective effect of uric acid, since uric acid levels were not directly analyzed in these studies. It remains to be seen whether this represents a definitive causal relationship.

In addition to the cohort study, there were more retrospective and cross-sectional analysis. Boccardi et al. did a retrospective study of UA and late-onset AD. A total of 232 subjects were divided into three groups, including healthy controls (HC), mild cognitive impairment (MCI) group, and $\mathrm{AD}$ group ( $n=65,95$, and 72 , respectively). SUA levels were significantly lower in the $\mathrm{AD}$ group $(4.84 \pm 1.30$ $\mathrm{mg} / \mathrm{mL})$ than in the healthy control group $(5.82 \pm 1.76$ $\mathrm{mg} / \mathrm{mL} ; P=0.001)$. After adjusting for age, sex, body mass index, and creatinine levels, the correlation that existed between SUA and AD showed that the level of SUA was independently relevant to the diagnosis of $\mathrm{AD}[10]$. The results showed that AD patients had a decreased level of SUA. UA may have a protective effect on elderly $\mathrm{AD}$ patients. Gonzalez-Dominguez et al. investigated metabolic differences in serum of $\mathrm{AD}$ patients and healthy controls by using gas chromatography coupled to mass spectrometry. As a result, SUA was markedly reduced in the AD group compared to 
HC [11]. The same finding has been found in four investigations in recent years [12-16]. However, in a cross-sectional analysis, Zuliani et al. concluded that UA levels of LOAD (late-onset $\mathrm{AD} ; 357 \pm 95 \mu \mathrm{mol} / \mathrm{L}$ ) were elevated compared to the control group $(300 \pm 96 \mu \mathrm{mol} / \mathrm{L} ; P<0.01)$ [17]. They concluded that a combination of markers including SUA may be a possible tool for the diagnosis of LOAD. In addition, three studies have reported increases or no change of UA in $\mathrm{AD}$ blood [18-20]. Observational evidence is susceptible to a large number of biases that limit causal inference, both residual confounding and reverse causation. It is required to conduct more studies to ascertain whether this is a causal relationship.

3.2. Results from Other Studies. Scholefield et al. systematically analyzed the results of various biochemical pathways in AD. The analysis showed the decrease of uric acid in biological fluids of $\mathrm{AD}$ cases and suggested that the decline in serum UA could be used as part of a generic biomarker for dementia [21]. In a meta-analysis, 21 case-control studies that included UA measurements were included to analyze plasma antioxidant status in $\mathrm{AD}$ patients and cognitively intact older adults. The result showed that plasma UA was markedly decreased in $\mathrm{AD}$ with a pooled mean difference (PMD) -27.37 $\mu \mathrm{mol} / \mathrm{L}$ (95\% CI: $-49.75,-5.00, P=0.02)$ compared to the control group [22]. The meta-analysis of Khan et al. also showed that the level of SUA in dementia patients was markedly decreased compared to $\mathrm{HC}$, especially in patients with $\mathrm{AD}$ [23]. Paradoxically, another analysis suggested that there was no significant difference in levels of SUA between $\mathrm{AD}$ patients and $\mathrm{HC}$, but with proper interpretation, there may be a trend toward decreased UA in AD [24]. The above analysis suggested that high uric acid could be relevant to low risk of $\mathrm{AD}$, which was also confirmed in an animal experiment. In an experimental study, Wang et al. observed differences in the expression of $\mathrm{AD}$ biomarkers (APP and BACE1) in rats with different serum uric acid levels. The results showed that rats in the high-SUA-level group had significantly lower protein levels of APP and BACE1 in the hippocampus [25].

Over the past few years, four two-sample Mendelian randomized analyses have been performed to investigate the relationship between genetically determined circulating UA levels and risk of AD. Mendelian randomization is a statistical method that uses genetic variants like single nucleotide polymorphisms as genetic tools to make causal inferences about the nature of exposure-outcome relationships [26]. Williams et al. extracted genotype-AD risk association statistics from data from a genome-wide association study of LOAD subjects $(n=17,008)$ and controls $(n=37,154)$ [27]. The result suggested that genetically determined UA was not associated with $\mathrm{AD}$ risk and higher exposure to UA does not reduce the risk of AD. Similar results could be found in the study of Efstathiadou et al. [26] and Yuan and Yang [8]. But interestingly, Wang et al. came to the opposite conclusion in the latest research in 2020. The study found that for every standard deviation, increase in levels of UA (1.33 $\mathrm{mg} / \mathrm{dL}$ ) was related to a 0.09 -fold increase in the risk of $\mathrm{AD}$ [28]. This analysis suggested that genetically predicted higher level of UA may lead to a higher risk of AD. The MR method prevents bias caused by reverse causality and minimizes bias caused by measured and unmeasured confounding factors. These findings can make up for shortcomings of causality in observational studies and enrich the existing evidence.

3.3. Summary of This Section. Among the related studies on SUA and the risk of AD, existing cohort studies and an animal experiment suggested that high SUA level indicated a low risk of $\mathrm{AD}$, and the evidence was stronger in women. Consistent with this, a large number of retrospective studies, cross-sectional analyses, and systematic meta-analyses showed that AD patients had lower SUA level than healthy controls. Conflicting conclusions of other studies may be due to the selection of subjects and the absence of subgroup analysis of dementia. Some Mendelian-random analysis indicated that gene-determined UA was not related to the risk of $\mathrm{AD}$, suggesting the importance of the influence of acquired confounders.

\section{Level of SUA Was Relevant to the Progression of AD}

4.1. Related Studies. With the progression of $\mathrm{AD}$, cognitive impairment becomes more and more serious. In recent years, only one cohort has observed cognitive decline in patients with $\mathrm{AD}$ at different baseline uric acid levels. Ye et al. used the AD Neuroimaging Initiative database to evaluate the cognitive decline of MCI and AD patients $(n=1064$, including $271 \mathrm{HC}, 596 \mathrm{MCI}$, and $197 \mathrm{AD})$. SUA and AD biomarkers in cerebrospinal fluid (CSF) were recorded once for baseline. Cognitive assessment scales were assessed continuously. The result showed that higher SUA level was related to slower cognitive decline, particularly in MCI and AD subgroups, and this association was more significant among female participants $(P<0.001)$. Cerebrospinal fluid biomarker models showed that high concentrations of UA attenuated the adverse effects of $\mathrm{A} \beta 1-42$ and tau on cognitive decline in female subjects. However, it was noteworthy that in male subjects, the interaction was limited to resistance to the adverse effects of $\mathrm{A} \beta 1-42$ only, and there was no significant interaction with tau [29]. These findings suggested that UA had protective actions against longitudinal decline of cognition and can interact with $\mathrm{A} \beta$ and tau. Higher level of SUA may indicate slower progression of $\mathrm{AD}$. The results also showed sex-related differences in uric acid's protective effects on cognition, which may partially explain the conflicting results and conclusions of the two cohort studies mentioned above.

This sex-related effect has also been observed in some other studies. A study of 1451 cognitively healthy adults found that elevated baseline SUA was relevant to decreased attention and visuospatial abilities in males. But in females, there were no marked findings [30]. A longitudinal cohort study of SUA and cognitive change showed that elevated level SUA at baseline was relevant to faster decline of cognition by using the visual memory/visuoconstruction ability test. UA only had a potential benefit for attention filed in older men [31]. Lin et al. found that the high level of SUA had different actions on spontaneous brain activities and cognitive 
TABLE 1: Latest prospective studies that assessed the relationship between SUA and AD.

\begin{tabular}{|c|c|c|c|}
\hline $\begin{array}{l}\text { First author, journal } \\
\text { year [Ref] }\end{array}$ & Population & Duration & Main results \\
\hline Scheepers et al. 2019 [4] & 1462 females & 44 years & $\begin{array}{l}\text { Lower risk of AD in women with higher SUA (HR 0.78; } \\
\text { CI } 0.66-0.91 \text { ). }\end{array}$ \\
\hline Latourte et al. 2018 [5] & 1598 individuals & 12 years & $\begin{array}{c}\text { Significant risk of VD or mixed dementia in patients with higher } \\
\text { SUA levels (HR 3.66, 95\% CI: } 1.29-10.41, P=0.015) \text {, compared } \\
\text { to AD (HR 1.55 (95\% CI } 0.92 \text { to } 2.61), P=0.10) \text {. }\end{array}$ \\
\hline Alam et al. 2020 [6] & 11,169 individuals & $\begin{array}{l}\text { 24.1-year median } \\
\text { follow-up period }\end{array}$ & $\begin{array}{l}\text { After adjustment including cardiovascular risk factors, SUA } \\
\text { and incident dementia showed an uncorrelated outcome } \\
(\mathrm{HR}, 1.03 ; 95 \% \mathrm{CI}, 0.88,1.21) \text {. Elevated baseline levels } \\
\text { of SUA were relevant to faster decline of cognition (25-year } \\
\text { global } z \text {-score difference, }-0.149 ; 95 \% \mathrm{CI},-0.246,-0.052) \text {. }\end{array}$ \\
\hline Cao et al. 2020 [7] & 502,528 individuals & $\begin{array}{l}\text { 8.1-year median } \\
\text { follow-up period }\end{array}$ & $\begin{array}{l}\text { People in the lowest level group of SUA had a } 25 \% \text { increased } \\
\text { risk of dementia compared to those in the highest SUA } \\
\text { level group (HR }=0.75,95 \% \text { CI: } 0.64-0.87) \text {. }\end{array}$ \\
\hline Ye et al.2016 [29] & $\begin{array}{l}1064 \text { subjects }(197 \mathrm{AD} \text {, } \\
596 \mathrm{MCI} \text {, and } 271 \mathrm{HC})\end{array}$ & $\begin{array}{l}\text { Mean duration } \\
2.9 \text { years }\end{array}$ & $\begin{array}{l}\text { Higher levels of uric acid were associated with slower } \\
\text { cognitive decline, particularly in the MCI and AD subgroups } \\
\text { and more prominently in female subjects }(P<0.01) \text {. }\end{array}$ \\
\hline
\end{tabular}

function in men and women [32]. This sex-dependent effect was also suggested in a cross-sectional analysis from the ELSA Brazil cohort [33].

Some scholars have pointed out that the conclusions of these studies may be due to the different standard SUA levels of men and women. Many studies have shown that women and men have different optimal SUA cutoff values for predicting metabolic syndrome, insulin resistance, cardiovascular status, the development of gout, etc. [34, 35]. Since the cutoff value for hyperuricemia in women is lower than that in men, at a certain high uric acid level, men and women actually have different elevations. If this had been taken into account in the data analysis, different results might have been found [36]. Honarpisheh et al. [37] also pointed out that sex was a biological variable in the pathological studies of neurodegenerative diseases. In future research, this factor should be considered in data analysis.

There were some studies on the influence of SUA on cognitive function, which were not $\mathrm{AD}$ targeted. In a crosssectional analysis, Xue et al. found that levels of SUA were markedly decreased in MCI patients $(292.28 \pm 63.71 \mu \mathrm{mol} / \mathrm{L})$ compared to HC $(322.49 \pm 78.70 \mu \mathrm{mol} / \mathrm{L} ; P<0.05)$. Markedly positive correlations were shown between the Minimental State Examination (MMSE) scores and levels of SUA $(P<0.05)$. UA was a protective factor for MCI (odds ratio $=0.999,95 \% \quad C I=0.987-0.999$ ) [38]. Similar results were found in a cross-sectional study of 10,016 individuals in Beijing, China [39].

Wang et al. enrolled 12798 middle-aged and elderly people over 45 years old in the follow-up study of health and pension in China. They measured the baseline plasma UA level, and the cognitive function was evaluated by a variety of methods. The results showed that middle-aged and older Chinese with high UA levels at baseline had better cognitive function, but not with rates of cognitive decline [40]. Xu et al. found that lower serum UA levels were related to cognitive dysfunction and could serve as a potential predictor for VD [41]. In a cross-sectional study in South Korea, Kim et al. observed the association along SUA, AD brain changes, and cognitive impairment. 430 dementia-free elderly subjects were enrolled in this study. The results suggested that levels of SUA were markedly relevant to $\mathrm{AD}$-characteristic area cerebral glucose metabolism (AD-CM) and borderline associated with MMSE. AD-CM was the link of UA and cognitive measure scores. A decreased level of SUA was relevant to $\mathrm{AD}$-related cerebral hypometabolism [42]. In previous studies, few studies had included multimodal brain imaging to observe the relationship between uric acid and brain changes. Subsequent research is needed to prove whether this represents a causal relationship or not. And only after this relationship has been confirmed by more studies can experiments and clinical trials be designed from this point to using SUA as a therapeutic target.

In contrast, in a case-control study, higher levels of circulating UA were considered to be relevant to impairment of cognition in pharmacologically untreated elderly subjects [43]. In a two-center study of 180 elderly maintenance hemodialysis (MHD), Zhang et al. found that SUA levels were independently and negatively correlated with MMSE scores [44]. High SUA level may lead to impairment of cognition in the elderly MHD patients. A cross-sectional study from Japan showed that the high level of SUA was independently related to deterioration of cognition, and UA had an adverse influence on cognition [45].

4.2. Summary of This Section. Existing cohort studies have suggested that the high SUA level was associated with slower cognitive decline in $\mathrm{AD}$ patients, and high concentration of SUA could attenuate the effects of $A \beta 1-42$ and tau on decline of cognition in female subjects. This gender-related effect has also been observed in some other studies, which may be due to the fact that the standard SUA level of men and women is different. A large number of cross-sectional studies found that the elderly with high UA level had better cognitive function and SUA was considered to be a protective factor of MCI. The decreased level of SUA was relevant to AD- 


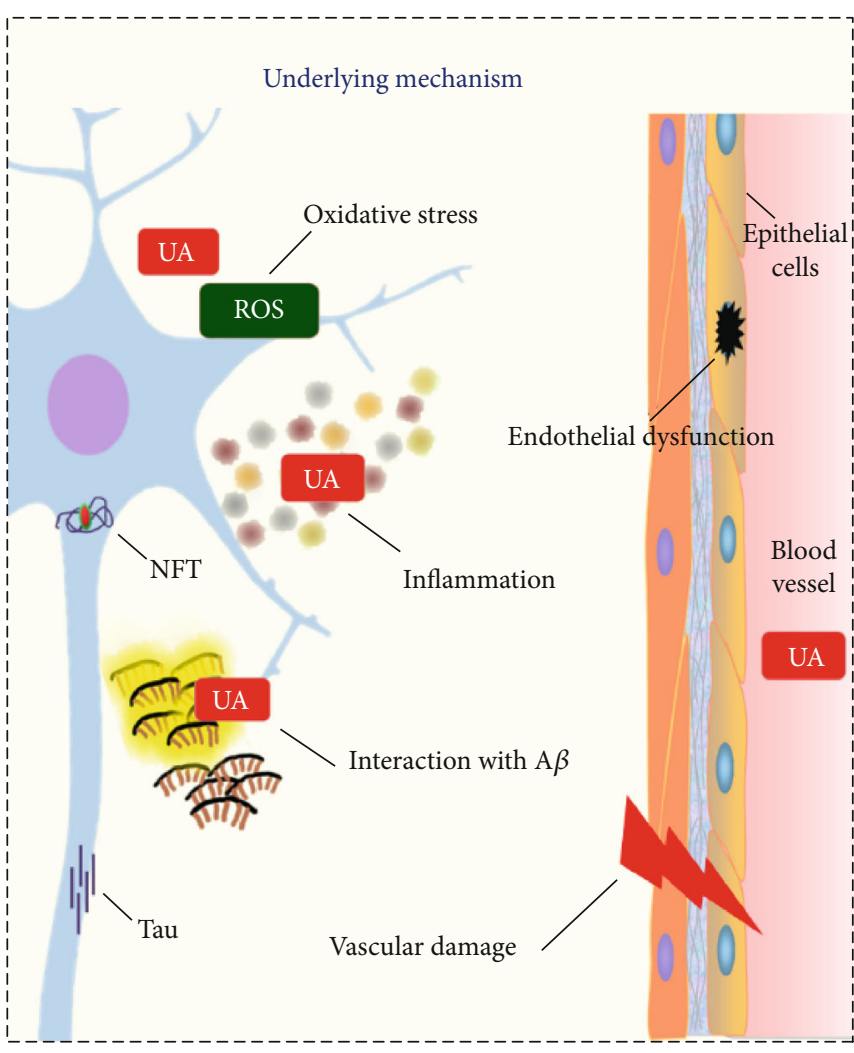

(a)

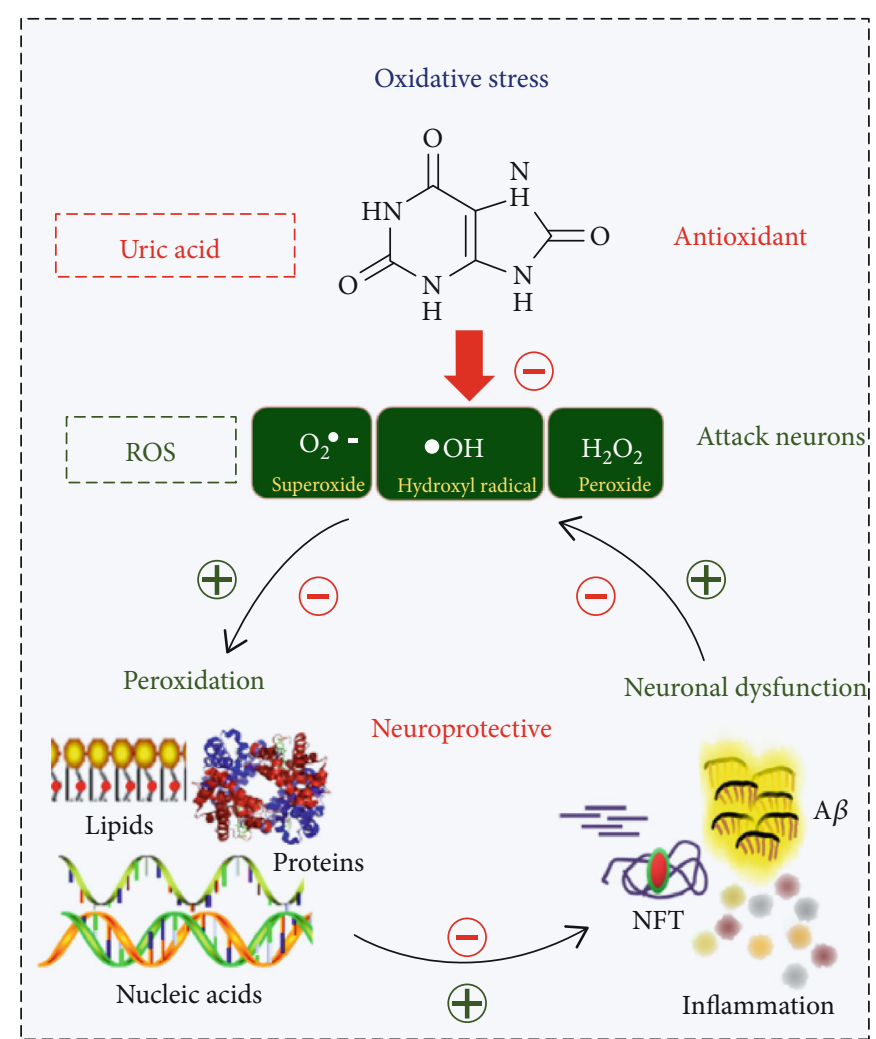

(b)

FIGURE 2: The underlying mechanism of uric acid on AD: (a) a summary figure on potential mechanisms; (b) SUA and AD were connected by oxidative stress.

related cerebral hypometabolism, but it is still uncertain whether there is a causal relationship. Still, some studies have shown that UA had an adverse influence on cognition (Table 1).

\section{UA Was Related to AD through Multiple Mechanisms}

Based on former research, UA had a dual effect on cognition including neuroprotective action and neurotoxic action. The dual effect of UA is reflected in a variety of hypotheses, including oxidative stress (OS), interaction with $\beta$-amyloid $(\mathrm{A} \beta)$, inflammation, endothelial dysfunction, and vascular damage [3].

Current research indicates that OS has a crucial part to play in these mechanisms. The influence of OS is particularly important in neurodegenerative diseases. The brain is especially vulnerable to reactive oxygen species (ROS). Lipids, proteins, and nucleic acids of neurons can be attacked by ROS, causing inevitable neuronal dysfunction [11]. Evidence showed that the brain tissue of $\mathrm{AD}$ patients is exposed to OS, which leads to peroxidation of lipid, protein, DNA, and RNA and glycoxidation [46]. These peroxidation products could promote the production of key pathological changes of $\mathrm{AD}$, including $\mathrm{A} \beta$, neurofibrillary tangles (NFT), and inflammation. In turn, pathological changes of $\mathrm{AD}$ could promote oxidative stress, which has been experimentally demonstrated in postmortem brain tissues in $\mathrm{AD}$ patients and $\mathrm{AD}$ transgenic mouse models [47]. Therefore, the two aspects interacted with each other and accelerated the development of $\mathrm{AD}$. Additionally, it has been demonstrated that increasing antioxidative potential correlated with a reduction of white matter injury [42].

UA is a natural and powerful antioxidant of humans. The antioxidant effect of UA is attributed to its capacity to chelate transition metal ions to make stable complexes [48, 49] and to act as a powerful cleaner of oxygen and hydroperoxyl radicals. The level of UA in cerebrospinal fluid was positively correlated with SUA level, especially when the blood-brain barrier was damaged [50]. Therefore, a higher serum uric acid level indicates a higher antioxidant capacity and may reduce the damage to the brain from oxidative stress. However, the antioxidant properties have also been challenged by some studies. Some scholars have suggested that under some specific conditions, uric acid even had an oxidationpromoting effect [51-53]. And there was insufficient data to analyze uric acid levels in the brain, and the limited available data did not suggest a significant change [54, 55] (Figure 2).

An in vivo experiment conducted by Shao et al. showed that UA induced hippocampal inflammation through the TLR4/NF- $\kappa$ B pathway and led to dysfunction of cognition. They also found that hyperuricemia in rats and people was relevant to gliosis in the hippocampus by using magnetic resonance imaging [56]. Another laboratory study showed that ITM2B was a regulator of GLUT9-mediated urate transport, which was a molecular link between UA homeostasis and 
neurodegenerative diseases [57]. Mazumder et al. [58] pointed out that uric acid was the most potent inhibitor of AChE, which was associated with dementia and cognitive impairment. In the Framingham Study, Chouraki et al. found the association with $\mathrm{AD}$ and hypoxanthine and taurine, which could be evidence that UA is neuroprotective [59].

Other potential links between uric acid and cognitive impairment can be suggested from the perspective of genetic syndromes and malnutrition. Some genetic syndromes are characterized by congenital uric acid disorders and neurological deficits, for example, primary renal hypouricemia [60, 61] and Lesch-Nyhan syndrome [62]. Therefore, it can be inferred that patients with the congenital uric acid disorder may have a genetic tendency to cognitive dysfunction. Since high uric acid levels increase cardiovascular risk, the relation between uric acid level and neurologic disorders could be like U- or J-shaped [61], which means if the UA level is extremely high or low, the risk of $\mathrm{AD}$ may both increase.

Hypouricemia is recognized as a sign of poor nutritional status, and some studies showed that poor nutritional status can cause faster cognitive decline in people with dementia [63]. There is a possibility that the correlation between low uric acid and cognitive impairment shown in the above study may include the influence of malnutrition. Cognitive frailty is a clinical syndrome in elderly individuals, which is characterized by physiological weakness and potentially reversible cognitive impairment, and dementia is excluded. The concept describes a preclinical cognitive status caused by physical frailty rather than neurodegenerative disorders [64]. The emergence of the concept of cognitive frailty supported this point. It provided a possibility that cognitive impairment may be caused by frailty or other comorbidities, and UA level is the manifestation of malnutrition, frailty, or other comorbidities rather than the main determinant.

\section{Conclusions}

The relationship between SUA and AD remains controversial, while current evidence supports the hypothesis that elevated UA levels could reduce the risk of $A D$, slow down the decline of cognition, and delay the progression of $\mathrm{AD}$, and high SUA level may related to lower risk and progression of AD. But findings of some studies contradicted it. The main reasons for contradiction between the results are study inconsistency, the difference in subject selection (sex and age), and the lack of subtype analysis of incident dementia.

The current studies have some shortcomings, such as only recorded the UA level at baseline, one-time record could not reflect long-term uric acid levels, the small number of large-sample, AD-targeted cohort studies, and inadequate adjustment of confounding factors like nutritional status and comorbidity.

Whether the dual effect of UA can be selectively controlled, whether it has different effects on men or women, and whether there is a causal relationship between low SUA and $\mathrm{AD}$-related cerebral hypometabolism are questions that need to be considered in the design of future studies. Future epidemiological studies should measure the SUA level several times to record dynamic changes and analyze the actual increase of SUA in different genders, carry out the assessment of nutrition, frailty, and comorbidity to fully exclude the influence of confounding factors, and combine imaging examinations in the study design.

\section{Conflicts of Interest}

The authors declare no conflicts of interest.

\section{Acknowledgments}

This work was supported by grants from the "Xing-lin Scholars" Project of Chengdu University of Traditional Chinese Medicine (No. QNXZ2018004), and "Hundred Talents Program" and Science and Technology Developmental Foundation from the Hospital of Chengdu University of Traditional Chinese Medicine (grant No. 20-Y02).

\section{References}

[1] P. Pacher, J. S. Beckman, and L. Liaudet, "Nitric oxide and peroxynitrite in health and disease," Physiological Reviews, vol. 87, no. 1, pp. 315-424, 2007.

[2] C. Tana, L. Busetto, A. Di Vincenzo et al., "Management of hyperuricemia and gout in obese patients undergoing bariatric surgery," Postgraduate Medicine, vol. 130, no. 6, pp. 523-535, 2018.

[3] C. Tana, A. Ticinesi, B. Prati, A. Nouvenne, and T. Meschi, "Uric acid and cognitive function in older individuals," Nutrients, vol. 10, no. 8, 2018.

[4] L. E. Scheepers, L. T. Jacobsson, S. Kern, L. Johansson, M. Dehlin, and I. Skoog, "Urate and risk of Alzheimer's disease and vascular dementia: a population-based study," Alzheimer's \& Dementia, vol. 15, no. 6, pp. 754-763, 2019.

[5] A. Latourte, A. Soumaré, T. Bardin, F. Perez-Ruiz, S. Debette, and P. Richette, "Uric acid and incident dementia over 12 years of follow-up: a population-based cohort study," Annals of the rheumatic diseases, vol. 77, no. 3, pp. 328-335, 2018.

[6] A. B. Alam, A. Wu, M. C. Power, N. A. West, and A. Alonso, "Associations of serum uric acid with incident dementia and cognitive decline in the ARIC-NCS cohort," Journal of the Neurological Sciences, vol. 414, p. 116866, 2020.

[7] Z. Cao, C. Xu, H. Yang et al., "Associations of BMI and serum urate with developing dementia: a prospective cohort study," The Journal of Clinical Endocrinology \& Metabolism, vol. 105, no. 12, 2020.

[8] H. Yuan and W. Yang, "Genetically determined serum uric acid and Alzheimer's disease risk," Journal of Alzheimer's Disease, vol. 65, no. 4, pp. 1259-1265, 2018.

[9] J. Y. Hong, T. Y. Lan, G. J. Tang, C. H. Tang, T. J. Chen, and H. Y. Lin, "Gout and the risk of dementia: a nationwide population-based cohort study," Arthritis research \& therapy, vol. 17, p. 139, 2015.

[10] V. Boccardi, S. Carino, E. Marinelli et al., "Uric acid and lateonset Alzheimer's disease: results from the ReGAl 2.0 project," Aging clinical and experimental research, vol. 33, no. 2, pp. 361-366, 2021.

[11] R. Gonzalez-Dominguez, T. Garcia-Barrera, and J. L. GomezAriza, "Metabolite profiling for the identification of altered metabolic pathways in Alzheimer's disease," Journal of pharmaceutical and biomedical analysis, vol. 107, pp. 75-81, 2015. 
[12] F. Mangialasche, M. Baglioni, R. Cecchetti et al., "Lymphocytic mitochondrial aconitase activity is reduced in Alzheimer's disease and mild cognitive impairment," Journal of Alzheimer's Disease, vol. 44, no. 2, pp. 649-660, 2015.

[13] E. Al-Khateeb, A. Althaher, M. Al-Khateeb et al., "Relation between uric acid and Alzheimer's disease in elderly Jordanians," Journal of Alzheimer's Disease, vol. 44, no. 3, pp. 859865, 2015.

[14] H. Hatanaka, H. Hanyu, R. Fukasawa et al., "Differences in peripheral oxidative stress markers in Alzheimer's disease, vascular dementia and mixed dementia patients," Geriatrics \& gerontology international, vol. 15, pp. 53-58, 2015.

[15] M. Cankurtaran, Y. Yesil, M. E. Kuyumcu et al., "Altered levels of homocysteine and serum natural antioxidants links oxidative damage to Alzheimer's disease," Journal of Alzheimer's Disease, vol. 33, no. 4, pp. 1051-1058, 2013.

[16] Z. Pu, W. Xu, Y. Lin, J. He, and M. Huang, "Oxidative stress markers and metal ions are correlated with cognitive function in Alzheimer's disease," American Journal of Alzheimer's Disease \& Other Dementias, vol. 32, no. 6, pp. 353-359, 2017.

[17] G. Zuliani, A. Passaro, C. Bosi et al., “Testing a combination of markers of systemic redox status as a possible tool for the diagnosis of late onset Alzheimer's disease," Disease markers, vol. 2018, Article ID 2576026, 9 pages, 2018.

[18] C. Cervellati, A. Romani, D. Seripa et al., "Oxidative balance, homocysteine, and uric acid levels in older patients with late onset Alzheimer's disease or vascular Dementia," Journal of the Neurological Sciences, vol. 337, no. 1-2, pp. 156-161, 2014.

[19] E. Trushina, T. Dutta, X. M. Persson, M. M. Mielke, and R. C. Petersen, "Identification of altered metabolic pathways in plasma and CSF in mild cognitive impairment and Alzheimer's disease using metabolomics," PloS one, vol. 8, no. 5, p. e63644, 2013.

[20] K. Nazef, M. Khelil, H. Chelouti et al., "Hyperhomocysteinemia is a risk factor for Alzheimer's disease in an Algerian population," Archives of medical research, vol. 45, no. 3, pp. 247250, 2014.

[21] M. Scholefield, R. D. Unwin, and G. J. S. Cooper, "Shared perturbations in the metallome and metabolome of Alzheimer's, Parkinson's, Huntington's, and dementia with Lewy bodies: a systematic review," Ageing Research Reviews, vol. 63, p. 101152, 2020.

[22] K. Mullan, C. R. Cardwell, B. McGuinness, J. V. Woodside, and G. J. McKay, "Plasma antioxidant status in patients with Alzheimer's disease and cognitively intact elderly: a metaanalysis of case-control studies," Journal of Alzheimer's Disease, vol. 62, no. 1, pp. 305-317, 2018.

[23] A. A. Khan, T. J. Quinn, J. Hewitt, Y. Fan, and J. Dawson, "Serum uric acid level and association with cognitive impairment and dementia: systematic review and meta-analysis," Age (Dordrecht, Netherlands), vol. 38, no. 1, p. 16, 2016.

[24] X. Chen, X. Guo, R. Huang, Y. Chen, Z. Zheng, and H. Shang, "Serum uric acid levels in patients with Alzheimer's disease: a meta-analysis," PLoS One, vol. 9, no. 4, article e94084, 2014.

[25] L. Q. Wang Junxia and X. Xinxing, "The effects of serum uric acid on expression of APP and BACE1 in rats," Chinese Journal of Nervous and Mental Diseases, vol. 2014, no. 8, pp. 479-482, 2014.

[26] A. Efstathiadou, D. Gill, F. McGrane, T. Quinn, and J. Dawson, "Genetically determined uric acid and the risk of cardiovascular and neurovascular diseases: a Mendelian randomization study of outcomes investigated in randomized trials," Journal of the American Heart Association, vol. 8, no. 17, p. e012738, 2019.

[27] D. M. Williams, S. Hagg, and N. L. Pedersen, "Circulating antioxidants and Alzheimer disease prevention: a Mendelian randomization study," The American Journal of Clinical Nutrition, vol. 109, no. 1, pp. 90-98, 2019.

[28] Z. Wang, L. Meng, L. Shen, and H. F. Ji, "Impact of modifiable risk factors on Alzheimer's disease: a two-sample Mendelian randomization study," Neurobiology of aging, vol. 91, pp. 167.e11-167.e19, 2020.

[29] B. S. Ye, W. W. Lee, J. H. Ham, J. J. Lee, P. H. Lee, and Y. H. Sohn, "Does serum uric acid act as a modulator of cerebrospinal fluid Alzheimer's disease biomarker related cognitive decline?," European journal of neurology, vol. 23, no. 5, pp. 948-957, 2016.

[30] A. M. Kueider, Y. An, T. Tanaka et al., "Sex-dependent associations of serum uric acid with brain function during aging," Journal of Alzheimer's Disease, vol. 60, no. 2, pp. 699-706, 2017.

[31] M. A. Beydoun, J. A. Canas, G. A. Dore et al., "Serum uric acid and its association with longitudinal cognitive change among urban adults," Journal of Alzheimer's Disease, vol. 52, no. 4, pp. 1415-1430, 2016.

[32] L. Lin, L. J. Zheng, U. Joseph Schoepf et al., "Uric acid has different effects on spontaneous brain activities of males and females: a cross-sectional resting-state functional MR imaging study," Frontiers in neuroscience, vol. 13, p. 763, 2019.

[33] C. P. Baena, C. K. Suemoto, S. M. Barreto, P. A. Lotufo, and I. Benseñor, "Serum uric acid is associated with better executive function in men but not in women: baseline assessment of the ELSA-Brasil study," Experimental gerontology, vol. 92, pp. 82-86, 2017.

[34] S. Seki, K. Tsutsui, T. Fujii, K. Yamazaki, R. Anzawa, and M. Yoshimura, "Association of uric acid with risk factors for chronic kidney disease and metabolic syndrome in patients with essential hypertension," Clinical and Experimental Hypertension, vol. 32, no. 5, pp. 270-277, 2010.

[35] M. L. Zhang, Y. X. Gao, X. Wang, H. Chang, and G. W. Huang, "Serum uric acid and appropriate cutoff value for prediction of metabolic syndrome among Chinese adults," Journal of Clinical Biochemistry and Nutrition, vol. 52, no. 1, pp. 38-42, 2013.

[36] P. Soysal, Comment on Serum uric acid is associated with better executive function in men but not in women: baseline assessment of the ELSA-Brasil study, vol. 96, p. 99, 2017.

[37] P. Honarpisheh and L. D. Mccullough, "Sex as a biological variable in the pathology and pharmacology of neurodegenerative and neurovascular diseases," British Journal of Pharmacology, vol. 176, no. 21, pp. 4173-4192, 2019.

[38] L. Xue, Y. Liu, H. Xue et al., "Low uric acid is a risk factor in mild cognitive impairment," Neuropsychiatric Disease and Treatment, vol. Volume 13, pp. 2363-2367, 2017.

[39] S. Xiu, Z. Zheng, S. Guan, J. Zhang, J. Ma, and P. Chan, "Serum uric acid and impaired cognitive function in communitydwelling elderly in Beijing," Neuroscience letters, vol. 637, pp. 182-187, 2017.

[40] T. Wang, Y. Wu, Y. Sun, L. Zhai, and D. Zhang, "A prospective study on the association between uric acid and cognitive function among middle-aged and older Chinese," Journal of Alzheimer's Disease, vol. 58, no. 1, pp. 79-86, 2017. 
[41] Y. Xu, Q. Wang, R. Cui, K. Lu, Y. Liu, and Y. Zhao, "Uric acid is associated with vascular dementia in Chinese population," Brain and behavior, vol. 7, no. 2, p. e00617, 2017.

[42] J. W. Kim, M. S. Byun, D. Yi et al., "Serum uric acid, Alzheimer-related brain changes, and cognitive impairment," Frontiers in Aging Neuroscience, vol. 12, p. 160, 2020.

[43] A. F. Cicero, G. Desideri, G. Grossi et al., "Serum uric acid and impaired cognitive function in a cohort of healthy young elderly: data from the Brisighella Study," Internal and emergency medicine, vol. 10, no. 1, pp. 25-31, 2015.

[44] J. Zhang, L. Tang, J. Hu, Y. Wang, and Y. Xu, "Uric acid is associated with cognitive impairment in the elderly patients receiving maintenance hemodialysis-a two-center study," Brain and behavior, vol. 10, no. 3, p. e01542, 2020.

[45] K. Suzuki, D. Koide, K. Fujii, T. Yamazaki, S. Tsuji, and A. Iwata, "Elevated serum uric acid levels are related to cognitive deterioration in an elderly Japanese population," Dementia and geriatric cognitive disorders extra, vol. 6, pp. 580-588, 2017.

[46] A. Gella and N. Durany, "Oxidative stress in Alzheimer disease," Cell adhesion \& migration, vol. 3, no. 1, pp. 88-93, 2009.

[47] C. M. Feitosa and O. G. L. Da Silva, "Determination of parameters of oxidative stress in vitro models of neurodegenerative diseases-a review," Current Clinical Pharmacology, vol. 13, no. 2, pp. 100-109, 2018.

[48] C. X. Santos, E. I. Anjos, and O. Augusto, "Uric acid oxidation by peroxynitrite: multiple reactions, free radical formation, and amplification of lipid oxidation," Archives of Biochemistry and Biophysics, vol. 372, no. 2, pp. 285-294, 1999.

[49] K. J. Davies, A. Sevanian, S. F. Muakkassah-Kelly, and P. Hochstein, "Uric acid-iron ion complexes. A new aspect of the antioxidant functions of uric acid," The Biochemical Journal, vol. 235, no. 3, pp. 747-754, 1986.

[50] G. L. Bowman, J. Shannon, B. Frei, J. A. Kaye, and J. F. Quinn, "Uric acid as a CNS antioxidant," Journal of Alzheimer's disease, vol. 19, no. 4, pp. 1331-1336, 2010.

[51] G. Desideri, R. Gentile, A. Antonosante et al., "Uric acid amplifies $\mathrm{A} \beta$ amyloid effects involved in the cognitive dysfunction/dementia: evidences from an experimental model in vitro," Journal of cellular physiology, vol. 232, no. 5, pp. 1069-1078, 2017.

[52] W. Lu, Y. Xu, X. Shao et al., "Uric acid produces an inflammatory response through activation of NF- $\kappa \mathrm{B}$ in the hypothalamus: implications for the pathogenesis of metabolic disorders," Scientific reports, vol. 5, article 12144, 2015.

[53] A. So and B. Thorens, "Uric acid transport and disease," The Journal of clinical investigation, vol. 120, no. 6, pp. 17911799, 2010.

[54] N. Mcfarland, T. Burdett, C. A. Desjardins, M. P. Frosch, and M. A. Schwarzschild, "Postmortem brain levels of urate and precursors in Parkinson's disease and related disorders," Neurodegenerative Diseases, vol. 12, no. 4, pp. 189-198, 2013.

[55] M. Zabel, A. Nackenoff, W. M. Kirsch, F. E. Harrison, G. Perry, and M. Schrag, "Markers of oxidative damage to lipids, nucleic acids and proteins and antioxidant enzymes activities in Alzheimer's disease brain: a meta-analysis in human pathological specimens," Free Radical Biology \& Medicine, vol. 115, pp. 351-360, 2018.

[56] X. Shao, W. Lu, F. Gao et al., "Uric acid induces cognitive dysfunction through hippocampal inflammation in rodents and humans," The Journal of Neuroscience, vol. 36, no. 43, pp. 10990-11005, 2016.

[57] A. K. Mandal and D. B. Mount, "Interaction between ITM2B and GLUT9 links urate transport to neurodegenerative disorders," Frontiers in physiology, vol. 10, article 1323, 2019.

[58] M. K. Mazumder, B. C. Phukan, A. Bhattacharjee, and A. Borah, "Disturbed purine nucleotide metabolism in chronic kidney disease is a risk factor for cognitive impairment," Medical hypotheses, vol. 111, pp. 36-39, 2018.

[59] V. Chouraki, S. R. Preis, Q. Yang et al., "Association of amine biomarkers with incident dementia and Alzheimer's disease in the Framingham Study," Alzheimers Dement, vol. 13, no. 12, pp. 1327-1336, 2017.

[60] I. Sebesta and B. J. P. Stiburkova, "Purine disorders with hypouricemia," Prilozi, vol. 35, no. 1, pp. 87-92, 2014.

[61] A. Latourte, T. Bardin, and P. Richette, "Uric acid and cognitive decline: a double-edge sword?," Current Opinion in Rheumatology, vol. 30, no. 2, pp. 183-187, 2018.

[62] S. Bell, I. Kolobova, L. Crapper, and C. Ernst, "Lesch-Nyhan syndrome: models, theories, and therapies," Molecular syndromology, vol. 7, no. 6, pp. 302-311, 2016.

[63] C. Sanders, S. Behrens, S. Schwartz et al., "Nutritional status is associated with faster cognitive decline and worse functional impairment in the progression of dementia: the Cache County Dementia Progression Study1," Journal of Alzheimer's disease, vol. 52, no. 1, pp. 33-42, 2016

[64] C. Chen, J. Park, C. Wu et al., "Cognitive frailty in relation to adverse health outcomes independent of multimorbidity: results from the China health and retirement longitudinal study," Aging, vol. 12, no. 22, pp. 23129-23145, 2020. 